\title{
Visually perceived motion in depth resulting from proximal changes. I
}

\author{
HANS MARMOLIN * \\ University of Uppsala, Svartbäcksgatan 10, S-753 20, Uppsala, Sweden
}

\begin{abstract}
According to a model for form and motion perception proposed by Johansson (1964), every two-dimensional change in the proximal stimulation is projected out as a motion in depth. The amount of perceived depth motion can then be predicted from the projective relationship between the proximal change and the projected motion. This prediction was tested in a series of experiments by using squares that continuously changed their sizes as stimuli, and measuring perceived distance of motion in depth. The obtained relationship between perceived and predicted distance of motion was curvilinear for all Ss. Furthermore, the majority of the Ss underestimated the motion systematically, the remainder overestimated it. Thus, the prediction given in the model could not be verified. However, an alternative projective relation based on the assumption that a fixed proportion of the change is not projected out as a motion but perceived as a change of size agreed quite well with the data, both with distance judgments and with judgments of perceived change of size.
\end{abstract}

Motions of objects in depth and/or changes of forms of objects are accompanied by continuous changes of the spatial pattern of light entering the eye. Geometrically, there exists no specific information in these patterns of changes, as they cannot at the same time specify motion and form changes of the objects. However, it has been shown that the visual system responds in a specific way to such stimulation (e.g., Braunstein, 1962; Johansson, 1964).

How does the visual system produce this specificity? A first answer to this question was given by Johansson (1964). In a series of experiments, he presented squares of light that shrank and grew in different complex ways. Johansson's experiments showed that the visual system decoded the proximal changes according to certain highly mechanical rules. The basic rule seemed to be to interpret every complex change in a proximal pattern (simultaneous change in two dimensions) as produced by a three-dimensional motion.

Johansson analyzed this relationship between the changing proximal stimulation and the percept in terms of a reversible projective system consisting of a stationary point (corresponding to the eye), a projective plane (the proximal stimulation), and a projective space (the perceptual space) (see Fig. 1). In this model, complex changes in the proximal stimulation are projected out as motions in the perceptual space. A basic assumption is, then, that the stimulus-percept relationship may be given in terms of projective geometry (see also Johansson \& Jansson, 1967; Johansson, 1971). Although this assumption, strictly speaking, is a quantitative one, it has not yet

*The author is indebted to Professor Gunnar Johansson for valuable discussion on this project and for his constructive criticism of the manuscript. Valuable assistance in conducting the experiments was provided by Gunilla Olsson. This investigation was made possible by grants to Professor Johansson from the Swedish Council for Science Research and the Tricentennial Fund of the Bank of Sweden. been tested by such experiments. Thus, the present study was designed to quantify the relation between the changing proximal stimulation and perceived motion in depth, and, more precisely, to test the specific projective formula for the stimulus-percept relationship given in the model.

The model describes how the visual system analyzes patterns with equal or different amounts of change in the $\mathrm{X}$ and $\mathrm{Y}$ dimensions (here referred to as regular and irregular patterns). Since, in an earlier study (Marmolin \& Ulfberg, 1972), some of the Ss did not perceive the irregular patterns exactly as the model predicts, the experiments to be reported here will be restricted to regular patterns.

According to the model, all change in a regular pattern should be projected out as a motion in depth. As a first approximation, Johansson assumes a linear relationship between a physical and a perceptual description of space. The formula for predicting the perceived distance of motion in depth from the proximal change is then specified to:

$$
\frac{Z}{K}=\frac{\Delta x}{X-\Delta x}
$$

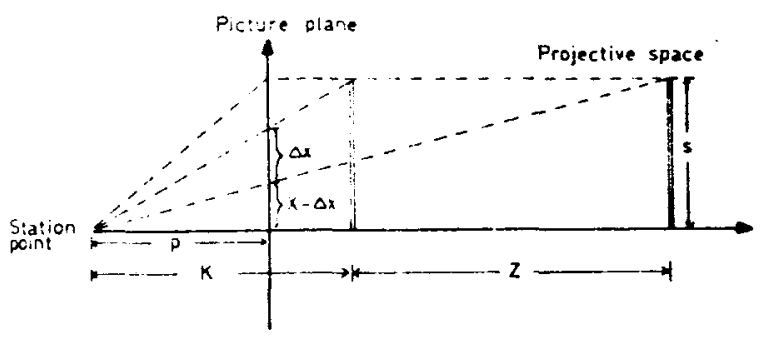

Fig. 1. The relation between amount of proximal change in the picture plane $(\Delta x)$ and the projected motion in the projective space $(Z)$ according to Eq. 1. In Fig. $1, X / p=s / K$ and $(X-\Delta \mathbf{x}) / \mathbf{p}=\mathbf{s} /(\mathbf{Z}+\mathbf{K})$, thus $Z / K=\Delta \mathbf{x} /(\mathbf{X}-\Delta \mathbf{X})$. 


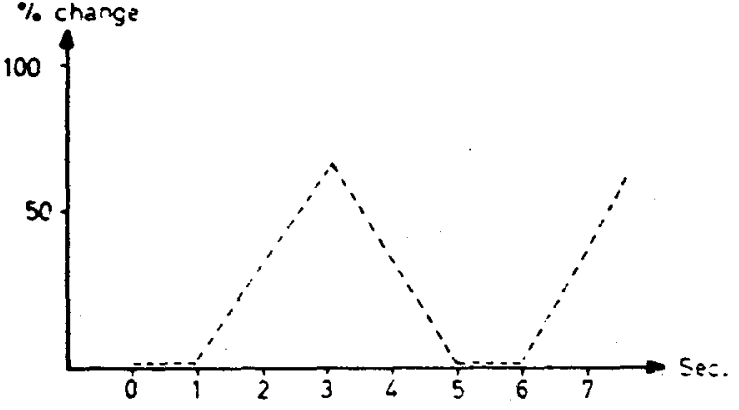

Fig. 2. The change of the contour of one of the patterns in Experiment 1.

In Eq. 1. $\mathrm{X}$ represents the initial size of the contour, $\Delta \mathrm{x}$. the amount of change (also expressed as percent change $\Delta x / X), K$. the perceived initial distance to the object, and $Z$, the amount of motion in depth (see Fig. 1). It should be noted that the model does not predict absolute distance of motion $(\mathrm{Z})$, only relative $(\mathrm{Z} / \mathrm{K})$.

Johansson also discussed the possibility of a curvilinear relation between a perceptual and a physical description of space of the form $R=S^{n}$, where $n \neq 1$. Equation 1 would then be transformed to

$$
\frac{\mathrm{Z}}{\mathrm{K}}=\left(\frac{\Delta \mathrm{x}}{\mathrm{X}-\Delta \mathrm{x}}\right)^{\mathrm{n}}
$$

The hypothesis about a curvilinear relation has some empirical support from experiments concerning changing stimulation (Marmolin \& Ulfberg, 1972), as well as static stimulation (Künnapas, 1960: Gogel. 1964: Gilinsky, 1951), but at the present stage of research, the data do not justify any definite conclusions.

In this context, it should be mentioned that Ittelson (1951) has conducted a series of experiments concerning perceived radial motion resulting from continuous changes of retinal size. From the transactionalistic point of view, Ittelson used "real" objects and studied determinants of both perceived amount of absolute and relative motion in depth. He demonstrated, among other things, that in monocular vision apparent movement is indistinguishable from real movement, that dynamic cues (i.e., proximal changes) in general override static cues, and that the perceived amount of relative motion depends on the relative amount of proximal change. Thus, these results support the model proposed by Johansson, but, since Ittelson used only $50 \%$ proximal change, the relation between perceived distance of motion in depth and amount of proximal change had to be studied further.

\section{A SCALE OF PERCEIVED DISTANCE OF MOTION IN DEPTH}

\section{Experiment 1}

The aim of Experiment 1 was to test the applicability of Eq. 1 or Eq. 2 by studying the relationship between amount of perceived relative motion in depth and amount of proximal change.

\section{Method}

Experimental technique. Stimuli were outlined squares (i.e.. only the contours were presented), which continuously shrank and grew (identical changes in the $X$ and $Y$ dimensions) with a constant rate of change, as Fig. 2 shows. These transformation patterns were generated by a digital computer (LINC-8), which plotted the squares on an oscilloscope in a darkened room. In order to avoid the perception of a screen carrying the patterns, the following technique was used. By means of an optical device. the patterns on the oscilloscope were projected out onto a translucent screen. This screen was placed between the oscilloscope and a collimator lens at focal distance to this lens. The Ss viewed the patterns (initial size of the contour. $5.5 \mathrm{deg}$ of visual angle) binocularly through the collimator lens. The binocular cues were, however. strongly reduced, as this lens gave parallel rays. i.e., identical visual images of the retina of the two eyes. The Ss were seated with their eyes directly at the lens. Otherwise. they perceived two different spaces, which was confusing.

Subjects. Experiment 1 consisted of three experiments. in which the perceived motion was measured during slightly differing conditions. Seven Ss participated in Experiment 1.1, six in Experiment 1.2. and five in Experiment 1.3. All but two of the $\mathrm{Ss}$ were ignorant of the hypothesis and had no experience with the experimental situation or the phenomena studied.

Procedures. The Ss estimated the magnitude of the relative motion $(\mathrm{Z} / \mathrm{K})$ by a straightforward technique similar to that of magnitude estimation. Ss task was to judge the ratio between the perceived motion in depth $(\mathrm{Z})$ and the perceived initial distance $(K)$. Hore sophisticated methods were tested in preexperiments. but were found to be unsuitable.

The computer presented the transformation patterns one by one in randomized order. and recorded the responses. The following procedures were used. A pattern was presented. and the $S$ judged the ratio between $Z$ and $K$. The inspection time was free, i.e., the Ss were allowed to take as long as necessary to judge the motions. Thereafter, the computer presented a number on the oscilloscope (a random number between 0.0 and 12.9). which the $S$ could adjust to a value corresponding to the estimated ratio, $Z / K$. The computer then recorded the response and presented the next pattern. The range of the numbers used had been determined by preexperiment. The Ss were told. however, that they could use larger numbers if necessary (just one $S$ did so on one occasion).

In Experiment 1.1. the computer presented 12 regular patterns with different amounts of change $(8.0 \%, 14.0 \%, 20.5 \%$. $31.5 \%, 51.6 \%, 62.5 \%, 67.0 \%, 70.5 \%, 73.5 \%, 76.5 \%, 79.5 \%$ and 87.5 change); in Experiments 1.2 and 1.3. there were $9(33.0 \%$, $50.0 \% 62.5 \% 72.0 \%, 76.5 \% 79.5 \% .84 .5 \% 87.5 \%$ and $98.5 \%$ change). Two replications were made of each stimulus in Experiment 1.1. three in Experiment 1.2. and six in Experiment 1.3.

\section{Results and Conclusions}

There were large individual differences in the amount of perceived relative motion. The Ss formed two different distributions, one low-scoring group (Group A, $13 \mathrm{Ss}$ ) and one high-scoring group (Group B, $5 \mathrm{Ss}$ ), as Table 1 shows. These two groups will therefore be treated separately. The interindividual variation was large also within the two groups (Table 1). The proximal 
Table 1

Judged $Z / K$ for the Different Transformation Patterns of Experiment 1

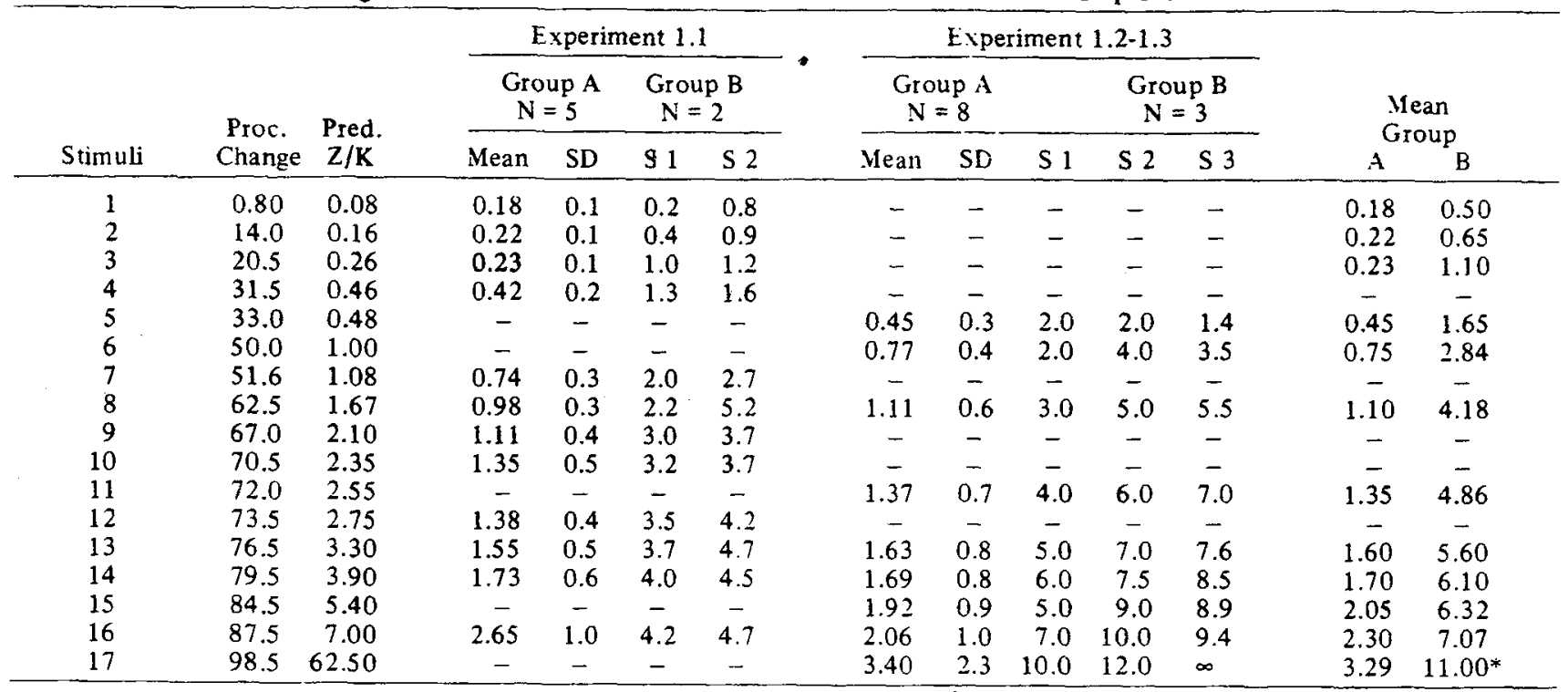

Note-Means and SDS of individual means are given. In the mean function, the values for the first three patterns are those of Experiment 1.1. the other means over Experiment 1.1 (interpolated), 1.2. and 1.3. Predicted $Z / K$ is calculated according to Formula 1 .

* 3 in Experiment 1.3 is not included.

change corresponding to judged $\mathrm{Z} / \mathrm{K}=1$ varied, for example, in Group A from about $30 \%$ to $90 \%$ change. However, there was almost no difference between the results of Experiments 1.1-1.3 with respect to the means over Ss for Group A. The three experiments will therefore be treated as one.

Figure 3 (Table 1) shows the obtained relation between the judged $Z / K$ and the theoretical, calculated according to Eq. 1. The relationship is curvilinear for both groups. Group A systematically underestimated the motion (except for changes less than 20\%) and Group B overestimated it. All Ss in the two groups conformed to these trends. One then had to conclude that the obtained judgments of perceived relative motion cannot be predicted from Eq. 1. This conclusion holds also for Eq. 2, as the log-log relation between judged and theoretical relative motion is not of the form $y=a x$ (Fig. 4). It should, however, be mentioned that the relation is approximately linear for limited relative distances.

There are at least three possible ways of explaining the deviations from the model. (1) Invalid scales of depth may have been obtained. (2) Some assumptions behind the derived formulas may not be applicable. (3) The visual system of man may not function according to the projective relationships. The aim of the remaining experiments in this report was to examine these three hypotheses.

\section{VALIDATIONS OF THE OBTAINED SCALES}

In order to be able to draw valid conclusions from the results of Experiment 1 about the projective equation of the model, the following assumptions are made: (1) The scales of motion in depth are ratio scales, the scale unit

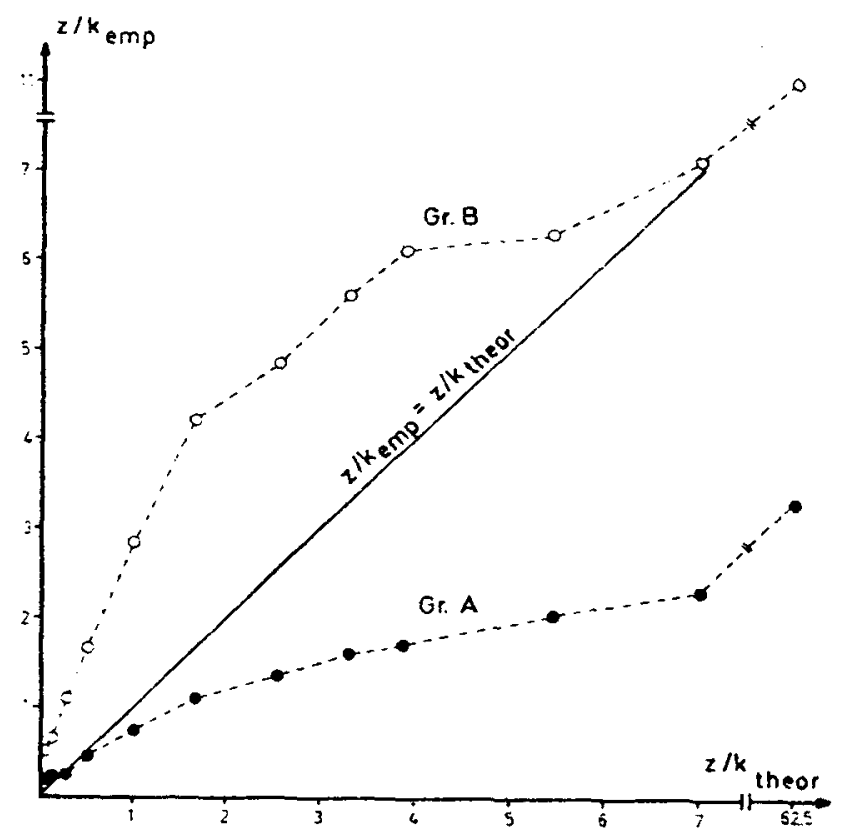

Fig. 3. For Groups $A$ and $B$, the relation between judged $Z / K$ ( $Z / K$ emp) and theoretical $Z / K(Z / K$ theor). Theoretical $Z / K$ is calculated according to $\mathrm{Eq}$. 1 . This figure is based on the first three transformation patterns used in Experiment 1.1 (means of individual means in Experiment 1.1 are plotted) and the nine patterns used in Experiments 1.2 and 1.3 (means of individual means in Experiments 1.1, 1.2, and 1.3 are plotted). The values from Experiment 1.1 were read off from the mean function obtained in this experiment. 


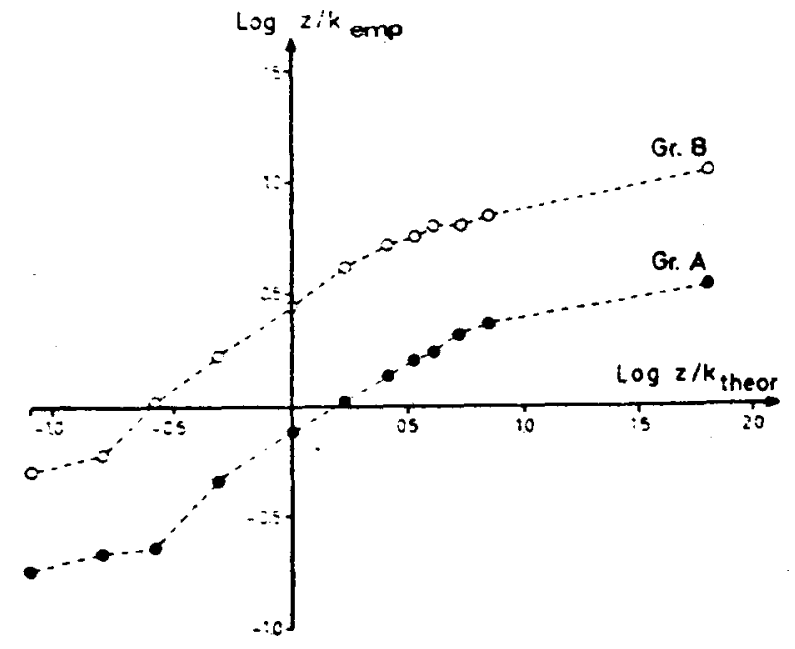

Fig. 4. For Groups $A$ and $B$, the $\log -\log$ relation between judged $Z / K(\log Z / K$ emp) and theoretical $Z / K(\log Z / K$ theor $)$. Theore tical $\mathrm{Z} / \mathrm{K}$ is calculated according to $\mathrm{Eq}$. 1 .

being perceived initial distance (K). (2) The Ss had really judged perceived motion. not amount of form change or time of motion. (3) There is no other information about depth available to the $S$ s other than the proximal change. These three assumptions were tested in Experiments 2. 3. and 4.

\section{Experiment 2}

In Experiment 1. it was assumed that the obtained scales were ratio scales expressed in $\mathrm{K}$ units. This assumption might, however, be questioned, since several of the methods used in preexperiments did not give ratio scales and since the large interindividual variation indicated that the Ss used different scale units.

According to Torgersson (1962), a scale can be regarded as a ratio scale if it possesses the following three properties: (1) The ratio of any two stimuli is independent of the values of the remaining stimuli in the series: (2) the calculated ratio between any two scale values agrees with the perceived ratio between the corresponding stimuli; and (3) equal differences in scale values correspond to subjectively equal intervals.

An experimental test of the ratio properties of the scales of Experiment 1 can be made by repeating the scaling using the method of equisection. This method does not require the $S$ s to estimate numerical values of sense ratios as does the method used in Experiment 1. The Ss have merely to decide if two distances of motion are equal or not. From such judgments, a ratio scale can be derived, and it can be expressed in $K$ units by measuring the perceived motion subjectively equal to the initial distance $(\mathbf{K})$. If this scale is identical with the one directly obtained in Experiment 1 , a reasonable conclusion would be that the two scales possess the three properties above and are expressed in $\mathrm{K}$ units.

The purpose of Experiment 2, then, was to get an equisection scale of perceived motion in depth in order to compare this scale with the scale of Experiment 1. In addition, the properties of the equisection scale were tested by repeating the experiment twice (Experiments 2.1 and 2.2) with different sizes of the interval to be sectioned (Torgerson, 1962).

\section{Method}

Experimental technique. By means of the computer. a square with $98.5 \%$ change was generated and presented to the $S \mathrm{~s}$. as in Experiment 1 (see Fig. 2).

In order to divide the perceived motion into distances of subjectively equal lengths. the following technique was used: As the square moved in depth, it could be stopped for about $0.5 \mathrm{sec}$ by pressing a response key at the appropriate moment. Then it stopped each time it passed this division point. The division point could be adjusted until it was made permanent by a second response key, and the corresponding amount of change was recorded by the computer. It was then possible to mark off a second division point, and so on, until the motion was divided into sections of equal length.

Subjects. Five $\mathrm{Ss}$. the same as in Experiment 1.3. participated in Experiment 2.1. Six new well-trained $\mathrm{Ss}$ participated in Experiment 2.2.

Procedure. The following procedures were used in Experiment 2.1. The regular pattern with a division point at 12.5\% change (D1) was presented for the Ss and they produced and adjusted a second division point (D2). so that the distance from the beginning of the motion to $D 1$ was perceived to be equal to the distance (D1-D2). Thereafter. the Ss marked off a distance (D2-D3) equal to (D1-D2), and so on, until the motion was divided into 10 subjectively equal distances. In addition. the Ss marked off a distance equal to the initjal distance, $K$. in a pattern without any division points. The $S$ s performed each of these two tasks three times.

Experiment 2.2 used a different standard distance, and the $\mathrm{S}$ did not successively divide the motion as in Experiment 2.1. The procedure was the following: The regular pattern with a division point at $37.5 \%$ change was presented for the $5 \mathrm{~s}$. who marked off a distance (D1-D2), as in Experiment 2.1. This was replicated five times. Thereafter, the pattern was presented with the two division points, D1 and the mean of D2, and the Ss marked off a third distance (D2-D3) five times. The pattern was then presented with the two division points, the mean of D2 and the mean of D3, and the Ss marked off a fourth distance, D4. five times. Finally, a distance corresponding to the initial $\mathrm{K}$ was marked off five times.

\section{Results and Conclusions}

A psychophysical magnitude function was derived for each $S$ relating percent change of the division points to perceived motion in depth. From these functions, perceived $\mathrm{Z} / \mathrm{K}$ corresponding to the stimuli used in Experiment 1.3 were read off, and the means of these readings were used in the analysis of data.

The individual differences were large in Experiment 2 also. The amount of change corresponding to the initial distance, $\mathrm{K}$, varied from $40 \%$ to $100 \%$ change. This complication will be discussed more closely later on. It should, however, be noticed that according to the results of Experiment 2.1 (Table 2), the individual differences seemed to be reproducible, at least for those who belonged to Group A. 
Figure 5 shows the relation between the equisection scale derived in Experiment 2.1 and the magnitude estimation scale obtained in Experiment 1.3 (as $\mathrm{S} 5$ belonged to Group B in Experiment 1.3, he is not included in the mean function). The two scales are almost identical for Ss 14. There are, however, systematic differences for $\mathrm{S} 5$. Also, the scale obtained in Experiment 2.2 agrees quite well with the scales for Group A obtained in Experiments 1 and 2.1.

On a prothetic continuum, a curvilinear relationship is often found between a category scale (as the equisection scale) and a magnitude scale (Stevens \& Galanter, 1957). With respect to this fact, the obtained close agreement between the two scales may be surprising. However, a closer look at Fig. 5 shows that there is a weak curvilinear tendency in the expected direction and, as Eisler $(1962 a, b)$ pointed out, the relation between a category scale and a magnitude scale may vary between an almost linear and a purely logarithmic one. It should also be mentioned that a plot of Gilinsky's (1951) equisection scale of static distance of depth against her ratio scale gives an approximately linear relationship.

The outcome of Experiment 2 thus indicates that the obtained scales of perceived relative motion, at least those of Group A, possess ratio properties and are expressed in K units. Group B data do not justify any strict conclusions, but the result of Experiment 2.1 points out that Group B may have given invalid judgments. As the scale of this group is linearly related to the scale of Group A (the two scales in Fig. 4 are parallel). the scale unit can be questioned. The judgments of Group B will be discussed further at the end of this report.

\section{Experiment 3}

In Experiments 1 and 2, the Ss' task was to judge the amount of perceived motion in depth, and they were carefully instructed not to judge time or amount of change. However, several Ss asserted that they had tried, but failed, to take an account of time.

In the method of equisection used in Experiment 2, the Ss' task was to divide the perceived motion in depth into subjectively equal distances. If these settings are more or less influenced by time of change, one would predict that a transformation pattern in which the rate of change was retarded would be divided into sections of successively greater lengths in comparison with a pattern with a constant rate of change. That is, the retarded pattern should produce less motion in depth.

Table 2

Amount of Change (Percent) Producing a Motion Corresponding to the Initial Distance in Experiments 1.3 and 2.1 for Ss 1-5 (Individual Means are Given)

\begin{tabular}{cccccc}
\hline Experiment & S 5 & S 4 & S 1 & S 2 & S 3 \\
\hline 1.3 & $<33$ & 33 & 50 & 85 & 94 \\
2.1 & 50 & 39 & 47 & 77 & 100 \\
\hline
\end{tabular}

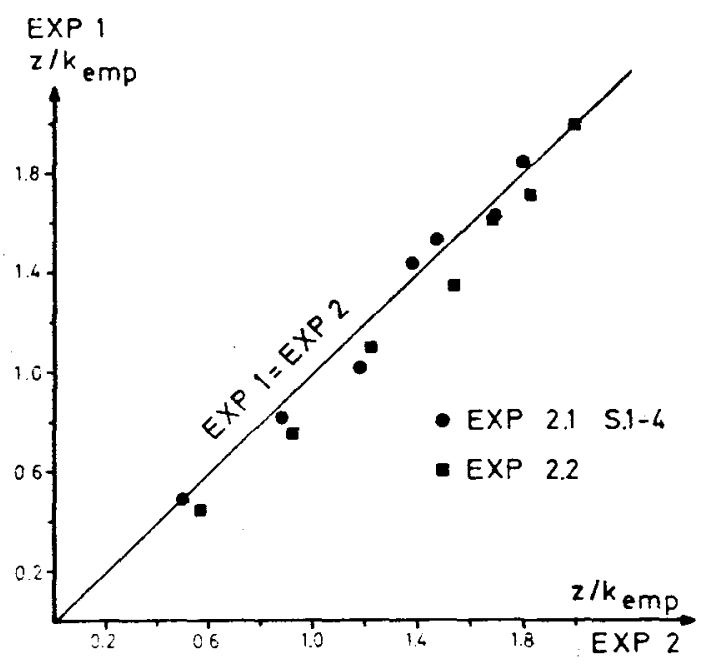

Fig. 5. The relation between derived $Z / K$ in Experiment 2.2 (means of individual means) and judged $Z / K$ for Group $A$ in Experiment 1 (means of individual means in Experiments 1.1, 1.2 , and 1.3 ), and the relation between derived $Z / K$ in Experiment 2.1 (means of individual means of $S s$ 1-4) and judged $Z / K$ for Group $A$ in Experiment 1.3 (means of individual means of Ss 1-4). S 5 is not included as he belonged to Group B in Experiment 1.3. S 5 obtained the following $Z / K$ values in Experiment 2.1: 1.0, 1.5, 2.0, 2.5, 2.75, 2.9, and 3.25, and the following in Experiment 1.3: 1.4, 3.5, 5.5, 7.0, 7.6, 8.5, and 8.9.

Experiment 3 was designed to test this prediction.

\section{Method}

The same experimental technique and method were used as in Experiment 2.1. The regular pattern. marked off at $37.5 \%$ change, was presented to the Ss, five students of psychology. who successively divided the motion into four sections of equal length. Thereafter. they marked out a distance equal to the initial distance. $K$. This was repeated four times for a pattern of constant rate of change $(21 \% / \mathrm{sec})$ and four times for a pattern with a linear retardation of the rate of change (the first $12.5 \%$ change took $0.5 \mathrm{sec}$ and the last, $1.0 \mathrm{sec}$; mean rate, $18 \% / \mathrm{sec}$ ).

\section{Results and Conclusions}

The mean settings of the division points were almost the same for the two patterns. The means for Division Points 1,2 , and 3 and the initial distance, $K$, were, for the retarded pattern, $39.9 \%, 49.3 \%, 55.5 \%$, and $39.1 \%$, and for the constant pattern, $39.5 \%, 50.4 \%, 56.7 \%$, and $39.1 \%$. An analysis of variance was made for each division point (mixed model) and, except for "between Ss," no significant effects resulted $(\mathrm{p}<.05)$. There may possibly be a very slight tendency to perceive a greater amount of motion in the retarded pattern, but clearly not less as predicted.

The conclusion from Experiment 3 must then be that the judgments of perceived motion in depth are not influenced by time of motion. Also, in this respect, the obtained scales seem to be valid.

There remains, of course, the possibility that the Ss had judged amount of change instead of motion in 
depth. However, there are several reasons to reject this hypothesis without testing it experimentally. Only one $S$ in Group B and none in Group A asserted that they had judged amount of form change: there is no linear relation between judged $\mathrm{Z} / \mathrm{K}$ and amount of change. This hypothesis was put forward in an earlier series of similar experiments (Marmolin \& Ulfberg, 1972), in which the Ss matched the motions of two transformation patterns, and was then disconfirmed. Besides, a critical experimental test in the type of situation used in this study is a very difficult, if not impossible, task.

\section{Experiment 4}

The purpose of Experiment 4 was to see if there were other sources of information about depth that could explain the obtained deviations from the model.

As outline figures were used. the effect of brightness ought to be negligible. However, the thickness of the contours might have had some effect. In Experiment 1 (and 2), the calculation of percent proximal change was based on the distances between the midpoints of the contours. If the Ss had responded to the outmost part of the contour instead. then there would be some underestimation. However, if predicted $Z / K$ is recalculated according to this assumption, then there would be just a slight change of the slope of the relation in Fig. 3. though not enough to explain the deviations from the model (the line would pass through the point $\mathrm{Z} / \mathrm{K}_{\text {theor }}=4.3 . \mathrm{Z} / \mathrm{K}_{\mathrm{emp}}=2.3$ ).

Another possible source of information, however, was available for the Ss. As mentioned above, the transformation patterns were viewed binocularly through the collimating lens system, which gives identical pictures at the retina of the two eves. For the Ss, this situation was more natural and not as strained as a monocular situation, but the binocular information was, strictly speaking, not eliminated, but "zero," implying no motion in depth. This information might then have counteracted the perceived relative motion in depth. Experiment 4 was designed to test this prediction.

\section{Method}

The same experimental technique. method, and procedure were used as in Experiment 3, but the two conditions were binocular and monocular judgment of a pattern with a constant rate of change $(21 \% / \mathrm{sec})$. After the experiment, the $S s$, six students of psychology, were questioned about how they experienced the two situations.

\section{Results and Conclusions}

According to the interviews, all Ss experienced the binocular situation as easier to judge and the square to be nearer in this situation than in the monocular one. However, the problem of this experiment is concerned with perceived relative motion, which was almost the same in the two situations. The mean of the division points, 1,2, and 3, and the initial distance, $K$, were, in the monocular situation, $40.2 \%$. $52.3 \%, 57.2 \%$, and $33.0 \%$, and in the binocular situation, $39.8 \%, 51.3 \%$. $57.5 \%$, and $34.7 \%$. An analysis of variance (mixed model, one analysis for each division. point) gave no significant effects except "between Ss" $(p<.05)$.

The conclusion therefore must be that the binocular situation was valid and can be used in experiments similar to those reported here.

\section{MEASUREMENTS OF PERCEIVED CHANGE OF SIZE}

The results of Experiments 2.4 gave no evidence that the deviations from the model were due to methodological factors. The second way of explaining the deviations was to question some assumption behind the two proposed equations. A basic assumption behind both is that all proximal change is to be projected out as a motion, and this may not be the case. For example. the underestimations should be explained if some fraction of change is not projected out.

As a first approximation, this fraction may be assumed to be a fixed proportion of the change (a). The stimulus-percept relationship will then be specified as

$$
\frac{Z}{K}=\frac{\Delta x-a \cdot \Delta x}{X-(\Delta x-a \cdot \Delta x)}
$$

Equation 3 has been fitted to the scale of Experiment 1 (Group A) by computing the value of a that gives a minimum of squared deviations. The agreement is almost perfect between predicted and perceived motion in depth, as Fig. 6 shows. According to Eq. 3, the individual differences can be described as a variation in a. The most appropriate experiment to test this hypothesis seems to be Experiment 2.2, and. as Fig. 7 shows, the correspondence between predicted and obtained motion in depth is also quite good for the individual scales.

There is, however, a slight systematic deviation from the theoretical line in Fig. 6 and especially in Fig. 7. It may be possible to account for this deviation by postulating a more complex relation between $\Delta \mathrm{x}$ and a (e.g., a threshold effect can be assumed). This seems, however, to be a rather unnecessary complication at this stage of research, as the agreement between theoretical and obtained $\mathrm{Z} / \mathrm{K}$ is good enough to make the hypothesis behind Eq. 3 plausible. The aim of Experiments 5 and 6 was to test this hypothesis in a more direct way.

\section{Experiment 5}

Experiment 5 was designed as a first. rather crude. 
test of the hypothesis discussed above. If correct, then the Ss ought to perceive some elasticity or corresponding changes of size in addition to the motion in depth. This prediction was tested in Experiment 5.1 by letting the Ss describe what they perceived when stimulated by a regular pattern, and in Experiments 5.2 and 5.3, by measuring a kind of detection threshold for perceived change of size.

\section{Method}

Subjects. Six Ss participated in Experiment 5.1, seven in Experiment 5.2, and rine in Experiment 5.3. All Ss in Experiment 5.1 were completely naive and did not know the purpose of the experiment. In Experiments 5.2 and 5.3, some of the Ss had participated in similar experiments.

Procedure. In Experiment 5.1. the computer generated and presented 15 regular transformation patterns (see Table 3 ) Preexperiments had shown that some Ss had a tendency to give size-change responses more often if this possibility was pointed out to them either by the instruction or by presenting very larges changes. Therefore, the patterns were presented just once. in a systematic order from small to large changes, and the Ss were instructed to describe all aspects of their percepts as naively as possible.

In Experiments 5.2 and 5.3, a kind of detection threshold was measured. A regular pattern with $98 \%$ change was presented, and the Ss who perceived any changes of size of the square or something corresponding to this were asked to mark off (as in Experiment 2) where in the motion they first detected the change of size. This was repeated three times in Experiment 5.2 and five times in Experiment 5.3.

\section{Results and Conclusions}

In Experiment 5.1, all Ss perceived changes of size of the squares (described in several different, often very imaginative, ways) in addition to motion in depth and changes of velocity. Of a total of 90 possible size respoñses, 30 were obtained. There were no such responses for patterns with small changes and there were individual differences in number of size responses (Table 3).

In Experiments 5.2 and 5.3, all Ss except two perceived a change of size of the squares. The detection threshold varied from about $40 \%$ to $75 \%$ change (Table 4). The Ss placed their marks with a rather high reliability, which indicates that the Ss really perceived a change of size. The mean SD for 11 Ss was $3.7 \%$ (two Ss had a SD of about $9 \%$ and one misunderstood the instruction). Three Ss who participated in both experiments obtained almost identical values in the two experiments $(51.1 \% \cdot 43.2 \%, 72.1 \%-71.3 \%$, and 49.0\%-50.3\% change).

In Johansson's experiments (Johansson, 1964) with similar transformation patterns, many Ss (most of the

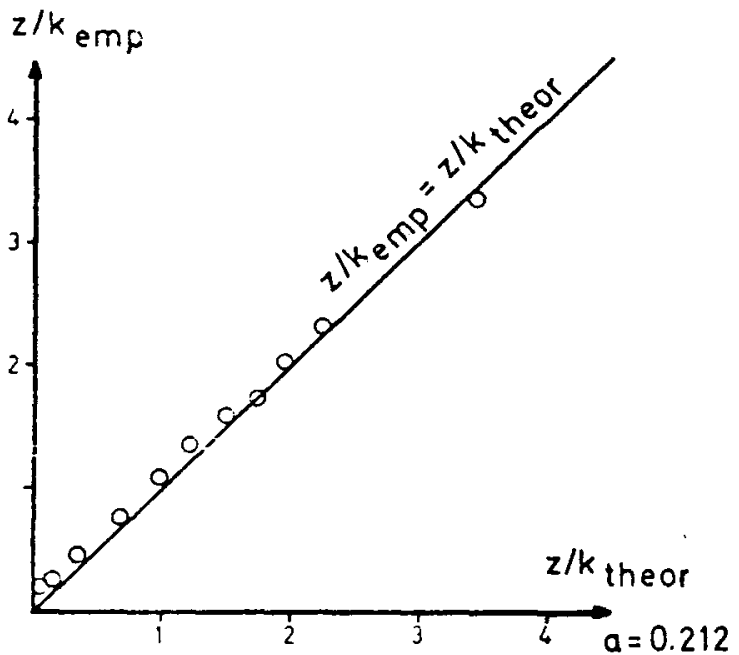

Fig. 6. For Group $A$ in Experiment 1, the relation between judged $\mathrm{Z} / \mathrm{K}(\mathrm{Z} / \mathrm{K} \mathrm{emp})$ in Experiment 1 (means of individual means in Experiments $1.1,1.2$, and 1.3 ) and theoretical $Z / K$ ( $Z / K$ theor). Theoretical $Z / K$ is calculated according to Eq. 3 .

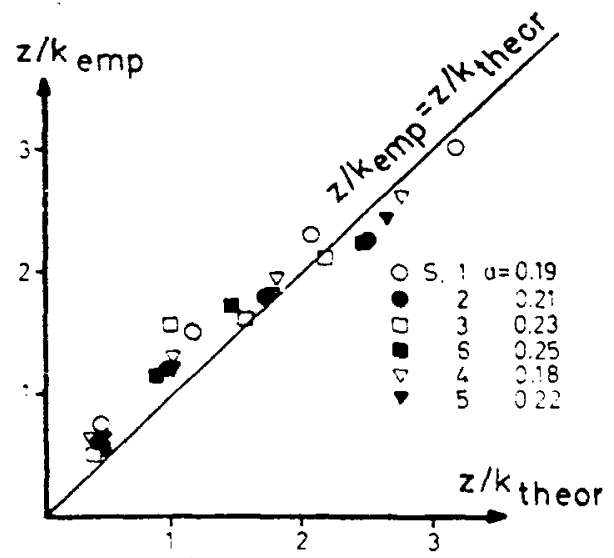

Fig. 7. For each $S$, the relation between derived $Z / K(Z / K$ emp) in Experiment 2.2 (means) and theoretical $Z / K(Z / K$ theor). Theoretical $Z / K$ is calculated according to Eq. 3 by, for each $S$, computing the value of a that gives a minimum of square deviations.

children and 19 of the 41 adults) also reported changes of size, but only when they were more closely questioned. Experiment 5 thus showed that the Ss perceived, in addition to motion in depth, a change in size of the squares.

\section{Experiment 6}

The purpose of this experiment was to reexamine, in a more critical way. the hypothesis tested in Experiment 5 . If it is to explain the deviations from the model, then there should be an interindividual negative

Table 3

Number of Percepts of Change of Size at Each Transformation Pattern in Experiment 5.1

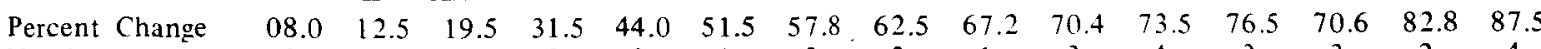

\begin{tabular}{lcccccccccccccc} 
Number & 0 & 0 & 0 & 2 & 3 & 1 & 2 & 3 & 1 & 3 & 4 & 3 & 3 & 2 \\
\hline
\end{tabular}


Table 4

Detection Threshold (Percent Change) for Perception of Change of Size for Each S and Replication in Experiments 5.2 and $5.3 *$

\begin{tabular}{|c|c|c|c|c|c|c|c|c|c|c|c|c|c|}
\hline & \multicolumn{5}{|c|}{ Experiment 5.2} & \multicolumn{8}{|c|}{ Experiment 5.3} \\
\hline & S 1 & $\mathrm{~S} 2$ & S 3 & 54 & 55 & $\$ 1$ & $\mathrm{~S} 2$ & S 3 & $\mathrm{~S} 4$ & 55 & S 6 & S 7 & $\mathrm{~S} 8$ \\
\hline $\begin{array}{l}M \\
\text { SD }\end{array}$ & $\begin{array}{r}58.7 \\
4.2\end{array}$ & $\begin{array}{r}51.5 \\
7.3\end{array}$ & $\begin{array}{r}75.6 \\
5.1\end{array}$ & $\begin{array}{r}72.1 \\
3.4\end{array}$ & $\begin{array}{r}49.0 \\
1.7\end{array}$ & $\begin{array}{r}75.6 \\
1.8\end{array}$ & $\begin{array}{r}72.6 \\
3.1\end{array}$ & $\begin{array}{r}69.1 \\
3.1\end{array}$ & $\begin{array}{r}71.3 \\
2.8\end{array}$ & $\begin{array}{r}58.4 \\
3.8\end{array}$ & $\begin{array}{r}43.2 \\
4.5\end{array}$ & $\begin{array}{r}50.3 \\
9.6\end{array}$ & $\begin{array}{r}48.8 \\
9.1\end{array}$ \\
\hline
\end{tabular}

*Ss 9 and 7 in Experiment 5.2 did not perceive anv change of size and $S 6$ in Experiment 5.3 misunderstood the instruction.

correlation between amount of perceived motion in depth and amount of perceived changes in size.

To directly measure the amount of perceived change of size in a valid way is a very difficult methodological task. However, the phenomenological description used in Experiment 5 can be translated into semiquantitative terms by assuming that the greater the change in size a $S$ perceives in comparison with other Ss, the more size responses would be given and the less amount of change is needed to perceive a change in the size of the squares.

In Experiment 6. then. the correlation between amount of perceived motion in depth and these two indicators of amount of perceived change of size was studied.

\section{lethod}

Subjects. Ten students of psychology participated as Ss. Eight of them had no experience in the experimental situation and were unaware of the purpose of the experiment.

Procedure. Experiment 6 consisted of two parts. In the first part. the amount of change producing a motion corresponding to the initial distance. $K$. Was measured. The same method was used as in Experiment 2. Five replications were made. This measure defined amount of perceived motion.

In the second part. 12 transformation patterns were presented once, as in Experiment 5 . in a systematic order from small to large changes $125 \%$ change. $31.25 \% 37.5 \%$ and so oni. the Ss describing how they perceived them. The number of patterns producing responses of elasticity. or something corresponding to this. and the smallest amount of change needed to produce such responses were measured. As stated above, these measures defined "amount" of perceived changes of size.

Table 5

Amount of Perceived Motion in Depth (Percent Change Corresponding to $Z / K=1$ ) and Amount of Perceived Change of Size (the Detection Threshold in Percent Change and the Number of Size Responses in Percent of the Total Number of Responses) for Each $S$ in Experiment 6

\begin{tabular}{cccc}
\hline $\mathrm{S}$ & $\begin{array}{c}\text { Percent } \\
\text { Size } \\
\text { Responses }\end{array}$ & $\begin{array}{c}\text { Detection } \\
\text { Threshold } \\
\text { Percent } \\
\text { Change) }\end{array}$ & $\begin{array}{c}\text { Peircent Change } \\
\text { Corresponding } \\
\text { to } Z / K=1\end{array}$ \\
\hline 1 & 0.6 & 50.0 & 81.4 \\
2 & 0.6 & 56.2 & 61.0 \\
3 & 0.5 & 56.2 & 62.5 \\
4 & 0.7 & 37.5 & 87.5 \\
5 & 0.3 & 68.8 & 48.5 \\
6 & 0.8 & 37.5 & 72.0 \\
7 & 0.4 & 62.5 & 57.8 \\
8 & 0.7 & 19.0 & 72.0 \\
9 & 0.4 & 62.5 & 62.5 \\
10 & Did not perceive any change of size \\
\hline
\end{tabular}

\section{Results and Conclusions}

The results are summarized in Table 5 . All Ss except one perceived a change in size in addition to motion in depth and changes in velocity. The rank correlation (Spearman) between amount of perceived depth motion and amount of perceived change in size. defined in the two ways as described above, was -0.84 and -0.80 (sign. $\mathrm{p}<.05$ ).

The outcome of Experiment 6 thus confirmed the prediction that the greater the amount of perceived motion in depth, the less the amount of perceived change in size. Together with the results of Experiment 5. these findings strongly support the assumption that with respect to those who underestimated the motion, the obtained deviations from the model are due to the fact that some fraction of change is not projected out as a motion in depth.

In addition, Experiment 6 showed that the individual differences in amount of perceived motion are not caused by methodological factors but are valid differences in the perception of motion in depth in the situation used.

\section{GENERAL DISCUSSION}

\section{Summary of the Experimental Results}

The problem of this investigation was to find out if perceived relative motion in depth could be predicted from Eqs. 1 or 2 as assumed by Johansson.

The outcome of the six experiments may be summarized as follows:

(1) Perceived relative motion in depth could not be predicted from Eq. 1 or Eq. 2. The relation between predicted and perceived relative motion was curvilinear. Furthermore, the majority of the Ss perceived considerably less motion than predicted. the remainder more. In addition, there were large interindividual differences in amount of perceived motion.

(2) These deviations from the proposed formulas could not be explained as being caused by methodological factors. The subjective scales possessed ratio properties and were expressed in such units $(\mathrm{K})$ as the analyses demanded (at least the scales of those who underestimated the motion); there was no tendency to judge time instead of motion; and the binocular information contained in the situation had no effect on perceived relative motion. 
(3) The basic assumption behind the two equations seemed to be partly invalid. According to the equations, all proximal change is projected out as a motion. However, the result of the experiments suggested that some fraction of change was not projected out as a motion but was perceived as a change in size. The obtained scales for those who underestimated the motion were consistent with Eq. 3; based on this hypothesis, almost all Ss perceived a change in size of the square in addition to the motion in depth, and there was an interindividual negative correlation between amount of perceived motion in depth and amount of perceived change in size.

\section{The Results Applied to the Model Investigated}

This investigation has shown that perceived relative motion cannot be predicted according to the equations of the model, and that the basic assumption behind the equations may be partly invalid. The model, therefore, would have to be modified in accordance with the result of this study if it is to allow quantitative predictions of perceived relative motion from changing proximal stimulation in situations like the one studied.

It can be argued that Eq. 2 of the model describes the distance judgments rather well over limited relative distances. However, the proposed alternative, Eq. 3, has a more general applicability with respect to the judgments of relative distance, and this equation agrees also with the obtained judgments of perceived changes in size. At this stage of research, Eq. 3, therefore, will be accepted as a more valid description of how the visual system analyzes proximal changes-at least in situations like the one used in this investigation.

A general assumption in the model is that the visual system is functioning according to the rules of projective geometry. As Eq. 3 is also based on projective geometry, this very basic assumption in the model has to be regarded as verified.

Another question of great importance for the model concerns the large interindividual differences found in this investigation, as in many others in this area of research (e.g., Marmolin \& Ulfberg, 1972; Johansson \& Jansson, 1968; Epstein, Johansson, \& Jansson, 1967; Epstein \& Park, 1963: Ittelson, 1951). A possible cause of this variation may be found in the impoverished situation, but this would indicate that there were other sources of information than the one postulated in the model necessary for correct distance perception. The interindividual variation cannot, then, be disregarded, and it complicates any explanation of the experimental results. The outcome of the experiments in this investigation indicates that the variation between those who underestimated the motion is valid, and it is possible for Eq. 3 to account for this variation by postulating different values of the constant. a. Eq. 3 is not as easily applied to those who overestimated the motion, but there are several facts which suggest that those Ss lack the ability to give valid judgments. Very few Ss gave suci responses ( 5 of 44 ), and they were not found when the easier and more natural method of equisection was used, no $\mathrm{S}$ perceived a growing object, as would be expected, and finally, underestimations are usually found in experiments like these (e.g., the references given above). This hypothesis, of course, has to be empirically tested before being fully accepted.

Accepting Eq. 3 as valid, the next question to be studied is, why is all change not projected out as a motion in depth? Several hypotheses are available, but only two will be mentioned.

First, the visual system may ordinarily function in that way. Systematic studies of amount of perceived motion in depth in a full-cue situation has to be carried out in order to answer this question.

Second, the change used in these experiments may not provide the visual system with enough or appropriate information about motion in depth. For example, the large changes of $90 \%$ and the constant proximal rate of change implicate a perception of an extremely high acceleration of the square if one is functioning according to the model (at 100\%, the velocity will be infinite). The visual system may therefore have solved this conflict in a rather consequential way by projecting out some change as a change in size.

\section{A Comparison with Other Scales of Distance in Depth}

There are some studies of static depth perception that have given scales of distance in depth that can be compared with the scales obtained in the present investigation.

Gilinsky (1951) studied the relation between perceived static distance in depth in an outdoor situation and physical distance and proposed a rational equation describing the distal stimulus-percept relationship. Equation 3 can be regarded as a proximal parallel to this equation. In Gilinsky's equation, perceived distance in depth (d) is predicted from physical distance (D) and perceived infinity (A) according to

$$
d=\frac{A D}{A+D}
$$

In Eq. 3, these notations correspond to

$$
A=\underset{\Delta x \rightarrow X}{\operatorname{Lim}} Z / K=(1-a) / a ; d=Z / K: D=\Delta x /(X-\Delta x)
$$

and Eq. 3 can then be reformulated to

$$
\mathrm{d}=\frac{\mathrm{AD}}{\mathrm{A}+\mathrm{D}+1}
$$

The importance of this close parallel has to be studied 
further as it may have implications for both Johansson's and Gilinsky's formulations. Künnapas (1960) has obtained scales of static distance in depth that is assumed to be related to physical distance by a power function of the form $\mathrm{R}=\mathrm{pS}^{\mathrm{n}}$. According to the results of the Künnapas study, the value of $n$ varies with the range of distances used in the experiment. The Smax $/ S \min$ ratio of 6 gave $n=1.47$, and the Smax $/ S \min$ ratio of $10, n=1.16$. Künnapas also fitted a power function to Gilinsky's data, and this gave an exponent of 0.67 for a $\mathrm{Smax} / \mathrm{Smin}$ ratio of 25 . If a power function is fitted to the data obtained in this investigation, a value of about 0.60 will be obtained (a straight line was fitted by eye to the points in Fig. 4, and the slope of this line was estimated). As the Smax/Smin ratio can be assumed to be about 60 (Smin is assumed to correspond to the initial distance), the scales obtained in this investigation support the conclusion reached by Künnapas about the dependence between degree of curviliniarity and stimulus range. It should, however, be noted that Gogel's (1964) data do not agree with this trend. Should the relation between perceived distance and physical distance be described by a power function as proposed by Künnapas and Gogel or by a rational equation like the one proposed in this report and by Gilinsky? As both kinds of relationships have given satisfactory descriptions of the data obtained, this question cannot be answered without systematic studies. However, the outcome of the present investigation indicates that a power function may be suitable for a rather limited stimulus range but not for a great one.

Finally, some comments should be made about the transactionalists' discussion of radial motion (Ittelson, 1951). Johansson assumes that the visual system automatically and directly decodes complex proximal changes as three-dimensional motions and/or form changes, but, according to Ittelson, changing retinal size becomes a cue to depth motion by means of an unconscious assumption about constancy of objective properties. There are, however, several experiments concerning static stimulation which indicate that this hypothesis may be unnecessary in order to explain the results (Epstein, Park, \& Casey, 1951; Gogel, 1964). Furthermore, the result of this investigation suggests that the visual system is able to simultaneously encode the same proximal change both as a movement in depth and as a change in size. If this result also holds for the same "real" objects that Ittelson studied, then the assumption about assumed object constancy can be seriously questioned with respect to motion in depth also.

\section{CONCLUSIONS}

In summing up this discussion, one has to conclude that the model proposed by Johansson has to be partly modified in correspondence with the result of this study. This investigation was restricted to regular patterns, but it should be possible to generalize to irregular patterns as well, as the same curvilinear relation between predicted and empirical motion in depth was found earlier for such patterns (Marmolin \& Ulfberg, 1972). In addition, this investigation shows that the model's geometrical approach to the problems of motion and form perception is applicable even in a quantitative way.

\section{REFERENCES}

Braunstein, M. L. Perception of depth through motion. Psychological Bulletin, 1962, 59, 422-433.

Eisler, H. Empirical test of a model relating magnitude and category scales. Scandinavian Journal of Psychology, 1962a, $3,88-99$.

Eisler, H. On the problem of category scales in psychophysics. Scandinavian Journal of Psychology, 1962b, 3, 81-87.

Epstein, W., Jansson, G., \& Johansson, G. Perceived angle of oscillatory motion. Perception \& Psychophysics, 1968, 3, 12-16.

Epstein, W., \& Park, J. Examination of Gibson's psychophysical hypothesis. Psychological Bulletin, 1964, 62, 180-196.

Epstein, W., Park, J. \& Casey, A. The current status of the size-distance hypothesis. Psychological Bulletin, 1961, 58, 419-514.

Gilinsky, A. S. Perceived size and distance in visual space. Psychological Review, 1951, 58, 460-482.

Gogel, W. Visual perception of spatial extent. Journal of the Optical Society of America, 1964, 54, 411-416.

Ittelson, W. Size as a cue to distance: Radial motion. American Journal of Psychology, 1951, 64, 188-202.

Johansson, $G$. Perception of motion and changing form. Scandinavian Journal of Psychology, 1964, 5, 181-208.

Johansson, G. Visual motion perception: A model for visual motion and space perception from changing proximal stimulation. Report No.98, Psychological Laboratory, University of Uppsala, 1971.

Johansson, G., \& Jansson, G. Perceived rotary motion from changes in a straight line. Perception \& Psychophysics, 1968, 4, 165-170.

Künnapas, T. M. Scales for subjective distance. Scandinavian Journal of Psychology, 1960, 45, 205-217.

Marmolin, H., \& U1fberg, S. Proximal changes and perceived distance of motion in depth. Report No. 125, Department of Psychology, University of Uppsala, 1972.

Stevens, S. S., \& Galanter, E. H. Ratio scales and category scales for a dozen perceptual continua. Journal of Experimental Psychology, 1957, 54, 377-411.

Torgerson, W. Theory and methods of scaling. New York: Wiley, 1962.

(Received for publication July 31, 1972; revision received February 12, 1973.) 